\title{
Why Expectations Will Determine the Future of Artificial Pancreas
}

\author{
Laurel H. Messer, RN, MPH, CDE
}

\section{Introduction}

A FTER MUCH ANTICIPATION, artificial pancreas (AP) technology is finally a commercial option for individuals with type 1 diabetes (T1D). AP is defined here as any system that automates the calculation of insulin delivery based on sensor glucose levels, from hybrid closed-loop therapy to future fully closed-loop or bihormonal systems. ${ }^{1}$ As companies move devices through the product development pipeline, options for people with T1D will likely increase exponentially in the upcoming decade.

There is little doubt that AP systems can improve glycemic control in individuals with T1D. A recent meta-analysis of 24 randomized controlled clinical trials of AP systems showed an overall $13 \%$ improvement in time in target range, and a 35min per day reduction in hypoglycemia. ${ }^{2}$ The Medtronic $670 \mathrm{G}$ pivotal trial, although uncontrolled, likewise demonstrated increased time in target range with $11 \%$ increase in adolescents and $7 \%$ increase in adults, with $35 \%$ and $46 \%$ decrease in hypoglycemia, respectively. ${ }^{3}$ While this oftenquoted statistics highlights the impact of AP, such data do not equip clinicians with meaningful information about who to recommend for AP therapy, or what to communicate to patients about AP practicalities. Many patients also utilize nonclinical online sources to satiate their curiosity of AP without the knowledge of their providers or diabetes educators. Closed-loop discussions, speculations, and recommendations are a constant on social media platforms, online diabetes forums, and within diabetes support groups. This is outside the control of the diabetes provider, but nonetheless magnifies the importance of the clinician's role: to be able to critically assess the appropriateness of AP therapy for an individual, educate competently on AP, and most importantly, set appropriate expectations for AP in the real world.

\section{Why Expectations Are Paramount}

Before the era of AP, expectations were noted to play a role in insulin pump satisfaction and continuance, ${ }^{4-6}$ continuous glucose monitoring (CGM) use, ${ }^{7,8}$ and sensor-augmented pump therapy. ${ }^{9}$ As diabetes technology advances in an upward trajectory, expectations take a more extreme course of highs and lows: with each new advancement, expectations soar with idealism and then fall, tempered by the reality of what that generation of technology can and cannot deliver. The buildup starts with marketing and spreads with innovators and early adapters who have higher risk tolerance and are more likely to overlook hassles of a new system for the sake of novelty. ${ }^{10}$ After more collective experience with these devices, a firm understanding of capabilities and limitations take shape, and expectations stabilize among clinicians and patients alike. Not surprisingly, it is in the upsurge and peak of expectations that the largest mismatch with reality exists, and patients are at their highest risk of discontinuing therapy due to disappointment and frustration they had not anticipated.

True-to-life expectations are paramount to the uptake and continuance of AP therapy, and are fortunately within the realm of clinician influence. There is theoretical precedent for the predictive power of expectations, described most simply in the technology acceptance model (TAM). ${ }^{11}$ The TAM was developed 30 years ago, conceptually derived from the theory of reasoned action, a well-established psychological/behavioral theory that helped explain volitional human behavior such as voting, exercise, and condom use. ${ }^{12}$ Davis and colleagues transformed these concepts into a technology-centric model, ${ }^{11}$ which has been adapted and applied across disciplines, from information technology and ecommerce ${ }^{13-16}$ to health science and medicine. ${ }^{17-20}$

The core constructs of the TAM are straightforward: "perceived usefulness" of a device and "perceived ease of use" of the device will lead to the behavioral intention to use the device. These constructs are familiar to diabetes providers and educators; however, we often use alternative language: the "benefits of technology" and the "burden of technology." These perceptions-or expectations - of AP devices inform the decisions of both clinician and patient. Van Bon et al. ${ }^{21}$ used the TAM as a framework to assess user expectations with future AP systems, also incorporating trust as a

CU School of Medicine, Barbara Davis Center for Diabetes, Aurora, Colorado.

The content within has been presented, in part, at the 11th International Conference on Advanced Technologies and Treatments for Diabetes (ATTD) in Vienna, Austria, February 17, 2018. 
central component. They found that $89 \%$ of individuals thought an AP would be useful, and $87 \%$ thought it would be easily handled. As this study was conducted many years ago, the results were based on hypothetical systems and do not reflect current expectations with AP. Nonetheless, the constructs were valid in pointing to behavioral intention to use a system.

\section{Mismatched Expectations with AP}

AP systems are not the optimal choice for all individuals with T1D, just as there is no "one size fits all" for diabetes care. When patients are persuaded to use AP based on unrealistically high expectations of usefulness and ease of use, they may experience dissatisfaction with the device that does not live up to the expectations. Discontinuation of AP due to frustration is disruptive to the clinical care of T1D, consuming valuable clinical resources and patients' time, money, and peace of mind. This can be largely avoided by increasing understanding of AP before device initiation.

One study investigated user expectations of AP after using a precommercial version of the $670 \mathrm{G}$ system in 32 individuals (ages 14-40 years) at 3 academic centers. ${ }^{22}$ Many of the subjects had high expectations of the AP before use. This included a perception that the device would be more "hands off," that they could eat unrestricted without glycemic excursions, or that the system would require minimal interaction. Others articulated more moderate expectations, understanding system burdens such as bolusing requirements and blood glucose checking. Interestingly, there was a key difference between these two groups. The group with mismatched high expectations had less knowledge about AP therapy on entry to the trial, whereas the subjects with more realistic expectations had more previous knowledge of AP than the high expectations group. The implications of this study are simple: when individuals are equipped with better information, they are more likely to set appropriate expectations on the perceived usefulness and ease of use of an AP system. According to the TAM, this will predict intention to use the technology, and likely continued use of the device. ${ }^{11}$

This has been observed in clinical care as well. As an example, at our large academic diabetes center, one 14-year-old adolescent asked to start the AP he had read about online that would take care of his $14 \%$ glycated hemoglobin $\left(\mathrm{HbA}_{1 \mathrm{c}}\right)$ while simultaneously eliminating all the work of diabetes: "no more checking, no more bolusing, it does it all." He believed that his problems with diabetes would be solved by AP within 6 months. Without serious readjustment of his unrealistic expectations, this patient would have become quickly frustrated and disappointed by the actual system performance and be at high risk for discontinuation. In contrast, other patients show a better understanding of insulin pumps and glucose sensors and ask nuanced questions about additional AP functionality and tasks. Still others express moderate hope in hypoglycemia protection due to their understanding of low suspend systems, while keeping an open mind about capabilities for hyperglycemia mitigation. Overall, the expectations are tied to experience and knowledge level.

\section{Clinician Responsibility to Set Appropriate Expectations}

If perceived usefulness and perceived ease of use (conventionally, benefits, and burden) are central to whether individuals embrace AP, diabetes clinicians have a crucial responsibility: to help set these expectations appropriately. This is done by as- sessing patients' knowledge of $\mathrm{AP}$ and equipping them with information reflecting the real potential gains and hassles of an AP system. Clinicians should ask unambiguous questions related to both usefulness (expected glycemic goals, glucose control, hopes for AP) and ease of use (learning curve, daily interaction with AP, troubleshooting), with more suggestions in the accompanying Figure 1. This discussion should commence when patients start to consider AP therapy, and continue through AP system initiation and throughout use. Here are potential points to consider when guiding expectations.

\section{Usefulness}

The usefulness of AP is largely in improved glycemic control. This is a realistic expectation for some people-those who struggle with overnight control, hypoglycemia, or persistent hyperglycemia. Studies with the current system show a reduction in $\mathrm{HbA}_{1 \mathrm{c}}$ of $0.5 \%$ in adults and $0.6 \%$ in adolescents after 3 months, while significantly reducing hypoglycemia and improving overnight glucose levels. ${ }^{3}$ Improved glycemic control is not equivalent to perfect control, however, and often the expectations for improvement can be off by an order of magnitude. A parent recently came to our clinic suggesting she may discontinue the AP system if her child continued to have glucose values $>140 \mathrm{mg} / \mathrm{dL}$ overnight. Her expectations needed significant resetting to understand the current state of the technology and reasonable goals for therapy. Furthermore, individuals who take scrupulous care of their diabetes may achieve better glycemic outcomes than those seen with current AP systems, making improvement an unlikely goal for a subset of users. ${ }^{2}$ Future systems will likely show greater improvement in glycemic control, as new algorithms may include customizable glucose targets, optional meal boluses, activity and sleep data, or incorporation of bihormonal therapy. For the current AP system and the future systems, consider carefully if AP may have glycemic benefit for your patient as you discuss the utility of AP.

Similarly, AP may be useful in improving quality of life for some, but not all people. AP can grant peace of mind to individuals who worry about hypoglycemia or have hypoglycemia unawareness by tangibly reducing occurrences. ${ }^{2,3,23}$ AP can also give users confidence that their glucose levels are being managed even when they are not actively making insulin dosing decisions. On the contrary, quality of life could be diminished by worries associated with AP. These might include concerns over whether the system is working, disagreeing with automated dosing, or finding ways to "trick" the system to perform differently. For individuals who prefer to be in complete control of all aspects of their diabetes care regimen, AP will likely bring disappointment and frustration. This includes the "Type A+" patients who will struggle to disengage from compulsive diabetes care and insulin decisions they cannot see (due to proprietary "black box" calculations) or change. Consider these patient characteristics when setting AP expectations, and assess ability or willingness to give partial control over to an AP system.

Finally, patients must understand the algorithm constraints of any AP system as they set expectations for usefulness. A quick survey of social media demonstrates individuals trading advice on letting the current $670 \mathrm{G}$ system "learn their body" or "learn their diabetes" as if the current AP algorithm considers mealtimes, exercise schedules, dietary choices, or diurnal hormone patterns. While future AP designs may do this, the current system 


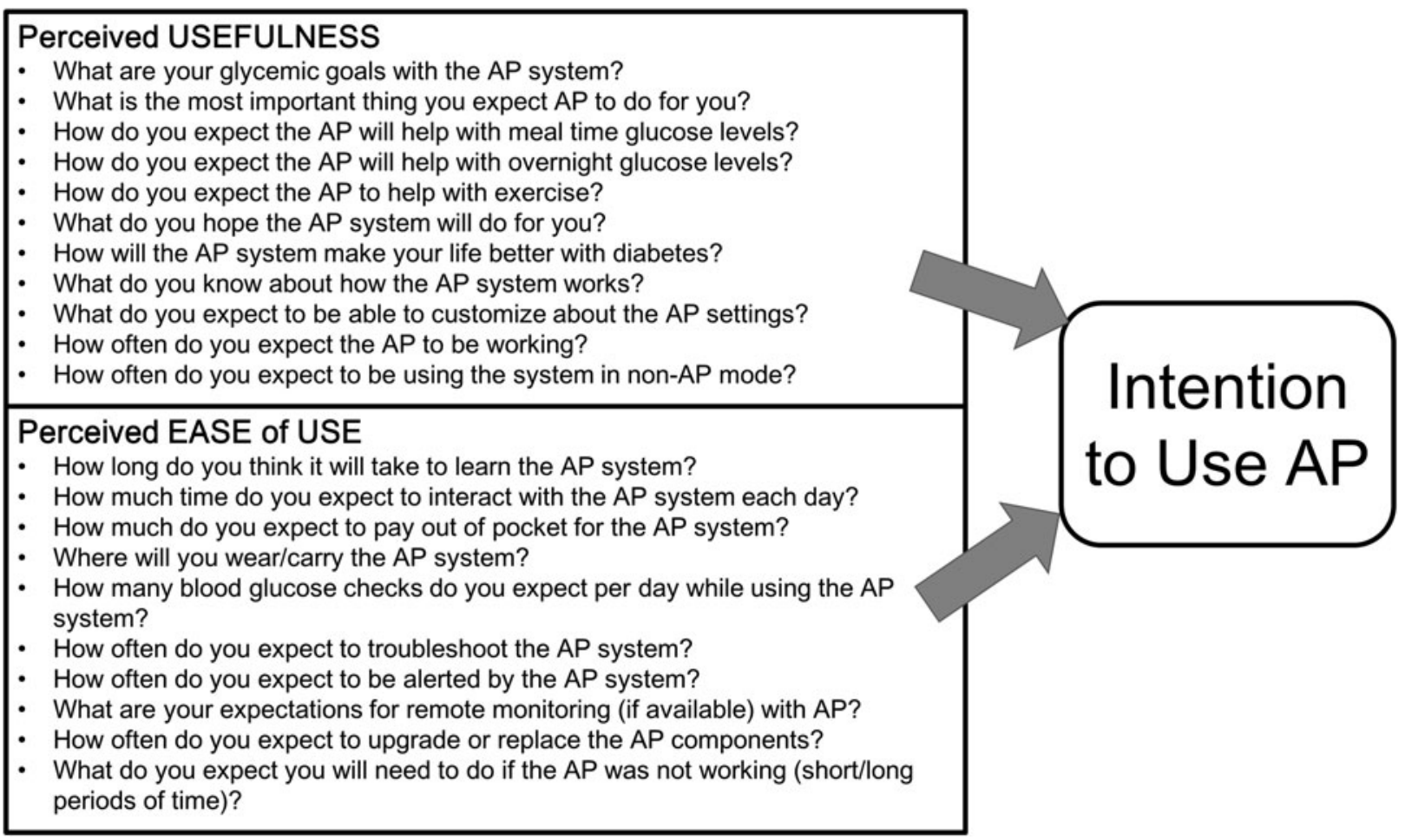

FIG. 1. Clinician-guided questions to assess patient expectations of Artificial Pancreas. $\mathrm{AP}=$ Artificial Pancreas.

only incorporates historical total daily dose to tune the algorithm. ${ }^{24,25}$ This is all that the system "learns." AP systems are likely to increase in sophistication and customization, even to the point of incorporating modular algorithms or dual hormone infusion with insulin and glucagon. Several patients at our clinic have expressed waiting for future systems that will include more customization of targets and algorithm parameters so that they are able to fine-tine and subsequently trust the system more. By understanding the general nature of AP intelligence, patients can adjust expectations for system adaptation over time. This will necessarily be different for every future system, prompting readjustment of expectations and education with each device. ${ }^{26}$

\section{Ease of use}

Patients considering AP need to understand the daily/ monthly/yearly maintenance of an AP system. Usability includes time and commitment required for training, cost of device, number of fingersticks needed, troubleshooting frequency, ability to customize and silence alerts, capability for remote monitoring, replacement frequency of components (both disposables and durable equipment), and adequacy of support. These will all factor into whether the system is perceived as easy to use. The current commercial system requires $2+$ calibration blood glucose levels per day, is not currently indicated to replace blood glucose checks for insulin dosing, requires regular troubleshooting to maintain operation, ${ }^{23}$ and does not facilitate commercial remote monitoring. This is a demanding trade-off considering other commercially available CGMs (not AP) are factory calibrated (requiring no blood glucose calibrations), last for 10-14 days, and require little user maintenance. The trade-off, of course, is worth it when there are tangible benefits to AP use.
Consider that some individuals may see reduced burden if they are used to intensively checking blood glucose levels (6-10 times per day) but are open to giving control over to the AP system. These individuals include the classic "type A" personalities, who are scrupulous with diabetes care but not as compulsive as previously mentioned "type A+" personalities. Patients currently using continuous subcutaneous insulin infusion or CGM may also already anticipate hassles associated with device wear, but it is unknown if this places them at an overall advantage when adapting AP therapy. TAM theory suggests that it will, and more studies are needed to explore this. Overall, individuals who anticipate the workload constraints of AP will likely adapt to the technology more easily than those who expect the system to run itself. ${ }^{22}$ Hopefully future AP systems will increase ease of use by eliminating CGM calibration (already a reality for some CGMs), reducing troubleshooting tasks, and increasing sensor duration. In the meantime, clinician-guided expectations will allow individuals to engage the system with eyes open and tolerate the hassles of use to gain better glycemic control or better quality of life.

\section{Conclusion}

There is still much to be learned about incorporating AP into clinical care. Future research will elucidate best practices, including appropriate indication for AP, patient selection characteristics, and optimal education strategies. All of these, however, will be contingent on provider and patient expectations of the system, setting the course for successful use.

The $670 \mathrm{G}$ has been on the market for 1 year, and clinicians and patients are better informed of what to expect, the rollercoaster wave of expectations having quelled. This is a place of progress for both clinician and patient. The next 
technological advancement will likely come soon, generating a new wave of high expectations and initial disappointments. As clinicians, we can prepare for this by cautiously tempering the expectations of our patients, orienting them to the likely reality of future advancements being incremental over the current reality. By setting appropriate expectations for the utility and ease of use for near-future devices, we place our patients at a significant advantage. Informed consumers of AP technology will likely be the ones to persist with the technology and reap benefits long term. As clinicians continue to learn who will be best candidates and appropriately recommend AP therapy, our success with AP will continue to improve. In the meantime, expectations reign paramount in patient intention to utilize AP technology.

\section{Acknowledgments}

The author acknowledges and thanks all of the BDC patients living with diabetes for their insights into using diabetes technology. Additional thanks to Gregory Forlenza, MD for reviewing this manuscript, and the entire BDC pediatric technology research team including Robert Slover, MD, Paul Wadwa, MD, Gregory Forlenza, MD, Cari Berget, Samantha Lange, Emily Jost, Lindsey Towers, Maninder Sethi, Katie Thivener, and Emily Westfall.

\section{Author Disclosure Statement}

L.M. is a contracted certified product trainer for Medtronic Diabetes, and has received speaking honoraria from Insulet Corporation and Tandem Diabetes Care.

\section{References}

1. Forlenza GP, Buckingham B, Maahs DM: Progress in diabetes technology: developments in insulin pumps, continuous glucose monitors, and progress towards the artificial pancreas. J Pediatr 2016;169:13-20.

2. Weisman A, Bai JW, Cardinez M, et al.: Effect of artificial pancreas systems on glycaemic control in patients with type 1 diabetes: a systematic review and meta-analysis of outpatient randomised controlled trials. Lancet Diabetes Endocrinol 2017;5:501-512.

3. Garg SK, Weinzimer SA, Tamborlane WV, et al.: Glucose outcomes with the in-home use of a hybrid closed-loop insulin delivery system in adolescents and adults with type 1 diabetes. Diabetes Technol Ther 2017;19:155-163.

4. Hofer SE, Heidtmann B, Raile K, et al.: Discontinuation of insulin pump treatment in children, adolescents, and young adults. A multicenter analysis based on the DPV database in Germany and Austria. Pediatr Diabetes 2010;11:116-121.

5. Ritholz MD, Smaldone A, Lee J, et al.: Perceptions of psychosocial factors and the insulin pump. Diabetes Care 2007;30:549-554.

6. Low KG, Massa L, Lehman D, Olshan JS: Insulin pump use in young adolescents with type 1 diabetes: a descriptive study. Pediatr Diabetes 2005;6:22-31.

7. Ritholz MD, Atakov-Castillo A, Beste M, et al.: Psychosocial factors associated with use of continuous glucose monitoring. Diabet Med 2010;27:1060-1065.

8. Hirsch IB: Clinical review: realistic expectations and practical use of continuous glucose monitoring for the endocrinologist. J Clin Endocrinol Metab 2009;94:2232-2238.
9. Rashotte J, Tousignant $\mathrm{K}$, Richardson $\mathrm{C}$, et al.: Living with sensor-augmented pump therapy in type 1 diabetes: adolescents' and parents' search for harmony. Can J Diabetes 2014;38:256-262.

10. Rogers E: Diffusion of Innovations. New York, NY: Free Press, 2003.

11. Davis FD: Perceived usefulness, perceived ease of use, and user acceptance of information technology. Manag Inf Syst Q 1989;13:319-340.

12. Fishbein M, Ajzen I: Belief, Attitude, Intention, and Behavior: An Introduction to Theory and Research. Reading, MA: Addison-Wesley, 1975.

13. Pavlou PA: Consumer acceptance of electronic commerce: integrating trust and risk with the technology acceptance model. Int J Electron Commun 2003;7:101-134.

14. Venkatesh V, Davis FD: A theoretical extension of the technology acceptance model: four longitudinal field studies. Manag Sci 2000;46:186-204.

15. Venkatesh V: Determinants of perceived ease of use: integrating control, intrinsic motivation, and emotion into the technology acceptance model. Inf Syst Res 2000;11:342-365.

16. Venkatesh V, Morris MG, Davis GB, Davis FD: User acceptance of information technology: toward a unified view. Manag Inf Syst Q 2003;27:425-478.

17. Holden RJ, Karsh BT: The technology acceptance model: its past and its future in health care. J Biomed Inform 2010; 43:159-172.

18. Kim J, Park HA: Development of a health information technology acceptance model using consumers' health behavior intention. J Med Internet Res 2012;14:e133.

19. Lai YH, Huang FF, Yang HH: A study on the attitude of use the mobile clinic registration system in Taiwan. Technol Health Care 2015;24(Suppl 1):S205-S211.

20. Udlis KA: The impact of technology dependency on device acceptance and quality of life in persons with implantable cardioverter defibrillators. J Cardiovasc Nurs 2013;28:E65-E73.

21. van Bon AC, Brouwer TB, von Basum G, et al.: Future acceptance of an artificial pancreas in adults with type 1 diabetes. Diabetes Technol Ther 2011;13:731-736.

22. Iturralde E, Tanenbaum ML, Hanes SJ, et al.: Expectations and attitudes of individuals with type 1 diabetes after using a hybrid closed loop system. Diabetes Educ 2017;43:223-232.

23. Messer LH, Forlenza GP, Sherr JL, et al.: Optimizing hybrid closed-loop therapy in adolescents and emerging adults using the MiniMed 670G System. Diabetes Care 2018;41:789-796.

24. Ruiz JL, Sherr JL, Cengiz E, et al.: Effect of insulin feedback on closed-loop glucose control: a crossover study. J Diabetes Sci Technol 2012;6:1123-1130.

25. Steil GM, Palerm CC, Kurtz N, et al.: The effect of insulin feedback on closed loop glucose control. J Clin Endocrinol Metab 2011;96:1402-1408.

26. Messer L, Jost E, Berget C, et al.: Automated insulin delivery (artificial pancreas) on the Horizon: educational considerations for youth with type 1 diabetes. J Pediatr Nurs 2017;34:104.

Address correspondence to: Laurel H. Messer, RN, MPH, CDE Barbara Davis Center for Diabetes 1775 Aurora Court, MS A140 (bldg M20 2233) Aurora, CO 80045

E-mail: laurel.messer@ucdenver.edu 